\title{
Pascale Hadermann, Ann Van Slijcke, Michel Berré (éds), La Syntaxe raisonnée.
}

Mélanges de linguistique générale et française offerts à Annie Boone à l'occasion de son $60^{\mathrm{e}}$ anniversaire. Préface de Marc Wilmet. Bruxelles : De Boeck - Duculot, 2003, 375 p.

\section{Carla Pellandra}

\section{(2) OpenEdition}

\section{Journals}

Édition électronique

URL : https://journals.openedition.org/dhfles/1285

DOI : $10.4000 /$ dhfles. 1285

ISSN : 2221-4038

Éditeur

Société Internationale pour l'Histoire du Français Langue Étrangère ou Seconde

Édition imprimée

Date de publication : 1 juin 2004

Pagination : 189-192

ISSN : 0992-7654

Référence électronique

Carla Pellandra, « Pascale Hadermann, Ann Van Slijcke, Michel Berré (éds), La Syntaxe raisonnée. », Documents pour I'histoire du français langue étrangère ou seconde [En ligne], 32 | 2004, mis en ligne le 01 janvier 2012, consulté le 27 mai 2021. URL : http://journals.openedition.org/dhfles/1285; DOI : https://doi.org/10.4000/dhfles.1285

Ce document a été généré automatiquement le 27 mai 2021.

(c) SIHFLES 


\section{Pascale Hadermann, Ann Van Slijcke, Michel Berré (éds), La Syntaxe raisonnée.}

Mélanges de linguistique générale et française offerts à Annie Boone à l'occasion de son $60^{\mathrm{e}}$ anniversaire. Préface de Marc Wilmet. Bruxelles : De Boeck - Duculot, 2003, 375 p.

\section{Carla Pellandra}

1 Les collègues et amis venus d'horizons et de pays différents pour cet hommage ont suivi des sentiers longtemps débroussaillés par Annie Boone : nous retrouvons ici plusieurs thèmes qu'elle a abordés au cours de sa longue carrière scientifique, thèmes qui pourraient être résumés par le titre qu'on a donné à ce recueil : c'est en effet avec une attention et une rigueur exemplaires qu'elle a toujours " raisonné » (et enseigné à raisonner) sur les faits linguistiques et notamment sur la syntaxe, à partir de sa thèse de doctorat portant justement sur les phrases complexes. Elle a animé récemment à Lyon, avec Brigitte Lépinette, Michel Berré et Pascale Hadermann une des journées d'étude de la SIHFLES sur «Syntaxe et enseignement des langues» dont les actes ont paru dans Documents n 29 de décembre 2002.

2 Ce beau livre s'ouvre sur une évocation affectueuse de la vie et de la carrière d'Annie et de son œuvre nourrie par les multiples approches méthodologiques qui ont caractérisé la pensée linguistique des dernières décennies, ses recherches s'orientant progressivement vers une approche sémantico-pragmatique. Nos lecteurs sont au courant de l'apport important d'Annie Boone à la connaissance de la tradition grammaticale belge et à l'histoire des grammaires françaises à l'usage des néerlandophones de Belgique que Michel Berré rappelle dans l'une des pièces liminaires.

3 Les éditeurs ont réparti les nombreuses contributions en quatre parties : Énonciation et pluralité discursive, Détermination et substitution, Connecteurs, prépositions et syntagmes prépositionnels, Enseignement du français: perspectives historiques et contrastives. On aborde des problèmes pointus et fascinants: du «double jeu du 
pronom on ", au sens du subjonctif, aux « référents évolutifs », aux multiples emplois de comme, à une analyse de tours à composante prépositionnelle, au complément de lieu... Je me bornerai à rendre compte de la dernière section plus proche de nos intérêts.

Trois articles sont consacrés à des analyses contrastives: Odile Halmøy se penche sur le gérondif, structure très fréquente en français, à l'oral comme à l'écrit, et sur les problèmes que pose sa traduction en néerlandais ou en d'autres langues germaniques qui n'ont pas de gérondif et en des langues romanes comme l'espagnol et l'italien, dont le gerundio ne couvre pas tous les emplois du gérondif français (p. 269-279). Dairine O'Kelly et André Joly font appel à la logique classique pour analyser les procédures de détermination du nom en irlandais, en anglais et en français. La distinction entre les opérations de définition, de classification, de description, et celles de détermination et de qualification, bref la distinction entre C'est un médecin et Il est médecin, bien mise en évidence en irlandais, est entièrement gommée en anglais... (p. 281-296). Kerstin Jonasson examine le traitement du discours narrativisé nominalisé (du genre raconter des histoires) dans deux versions suédoises de Une vie de Maupassant (p. 297-308).

Pascale Hadermann nous offre une analyse critique de la phrase complexe dans quelques grammaires scolaires de FLE publiées en Belgique entre 1920 et 1940. Ce n'est que dans deux grammaires pour les élèves avancés - publiées en 1925 et en 1937 qu'elle a repéré des analyses assez approfondies sur la phrase complexe. Tous les auteurs des grammaires insistent sur l'utilité d'une approche contrastive, approche contrastive qui consiste essentiellement dans l'emploi de quelques termes néerlandais pour quelques notions grammaticales. Une analyse ponctuelle des classements des subordonnées de ces deux grammaires révèle en effet que leurs deux auteurs ont été influencés surtout par l'évolution de la grammaire du français pour l'enseignement aux élèves francophones, donc par le deuxième courant de la grammaire scolaire française et par la Pensée et la langue de Ferdinand Brunot. Quant à la méthode, tout le monde préconise une approche inductive, mais il n'y a qu'une seule grammaire où les exemples précèdent l'exposé théorique... (p. 309-322). Brigitte Lepinette analyse la syntaxe dans une grammaire elemental y filosófica du français éditée en Espagne en 1868 (p. 323-337), une grammaire assez représentative de la doctrine syntaxique de son époque. Les grammaires du français remplissaient à l'époque un rôle de grammatisation des apprenants espagnols parce que la grammaire espagnole, enseignée dans un phase très précoce des études, n'était ensuite présente dans le cursus secondaire qu'en relation étroite avec l'enseignement du latin (situation qu'on retrouve dans beaucoup de pays où le latin constituait l'axe portant de l'enseignement linguistique), donc dans une perspective contrastive. L'auteur de cette grammaire filosófica, García de Modino, a la même exigence : sa doctrine syntaxique "reflète assez fidèlement l'évolution de la syntaxe scolaire en France »; elle permet donc d'initier les élèves aux théorisations grammaticales venant de France, mais la syntaxe qui avait été élaborée pour mettre au point les exercices scolaires d'analyse grammaticale et logique pour les petits Français devient en Espagne un catéchisme avec des définitions et des explications de grammaire générale, mais sans applications pratiques à l'enseignement du français qui continuera à être dispensé suivant l'approche contrastive traditionnelle (p. 323-337). C'est une approche contrastive analogue que Nadia Minerva a relevée dans la "vue d'ensemble " de son corpus de manuels publiés en Italie de 1861 à 1923 (qu'elle a récemment édité dans Insegnare il francese in Italia, Bologna, CLUEB, 2003) : un corpus constitué par des milliers de manuels où il n'y a qu'une vingtaine d'ouvrages 
entièrement consacrés à la syntaxe, syntaxe qui généralement figurait dans la dernière section des cours de français. Une approche contrastive abordée polémiquement par certains auteurs de manuels contre ceux qui resservent, sans aucun changement, leurs sources françaises «en enseignant beaucoup de syntaxe générale, peu de syntaxe française, aucune syntaxe comparée italienne française ». Une "syntaxe comparée " qui tourne toujours autour du stéréotype de la "clarté française", représentée essentiellement par la structure SVO, qui recommande d'éviter les longues périodes de l'italien écrit de l'époque (p. 339-353). Pierre Swiggers clôt cette promenade à travers les lieux élus de la recherche d'Annie Boone par une étude de l'apport fondamental fourni par le "grammairien patriote » Urbain Domergue à la théorie de la syntaxe, un apport particulièrement évident dans la quatrième édition de sa Grammaire françoise simplifiée élémentaire de 1791 , soit par son «innovation terminologique et classificatoire ", soit par la "mise en pratique de principes d'analyses harmonisant les deux aspects fondamentaux d'une langue: sémantisme grammatical et organisation syntaxique ». La grammaire de Domergue, malgré son nom, était destinée aux élèves aussi bien qu'aux maîtres de langue, d'où une attention pour les règles mises à la portée de l'intelligence "la plus commune ", règles à partir desquelles le maitre avisé devait remonter aux principes «transcendant les usages particuliers d'une langue ». Swiggers met bien en relief les mérites de Domergue dans l'élaboration d'une morphosyntaxe intégrée où se trouvent heureusement alliées la théorie de la grammaire et la pratique enseignante.

\section{AUTEUR}

\section{CARLA PELLANDRA}

Università di Bologna 